The Impact of Large-Scale Surveys on Pulsating Star Research

ASP Conference Series, Vol. 203, 2000

L. Szabados \& D. W. Kurtz, eds.

\title{
New Progress in Mode Detection and Identification in $\delta$ Scuti Stars by the Analysis of Line Profile Variations
}

\author{
L. Mantegazza, E. Poretti, M. Bossi, N. S. Nuñez, A. Sacchi, F. M. Zerbi \\ Osservatorio Astronomico di Brera, Via E. Bianchi 46 I-23807 Merate, \\ Italy
}

\begin{abstract}
Sct stars are among the most promising targets to perform ground-based asteroseismology. High resolution spectroscopy offers us a powerful technique to identify radial and nonradial pulsation modes, since we can easily detect oscillations and travelling features in the line profiles.
\end{abstract}

The developments of many studies, both observational and theoretical, are proving that the presence of a rich mixture of simultaneously excited radial and nonradial $p$ modes is quite common among $\delta$ Sct stars. In the recent years our group has focussed observations on a few stars already observed in the past, in particular FG Vir, BV Cir, X Cae and BB Phe. We devoted six campaigns to them, collecting 1065 spectrograms in 295 observing hours; in some cases we also performed simultaneous photometric measurements. Each star has been observed in two different campaigns, so that it has been possible to make a comparison between two independent datasets, and then check for possible variations in the pulsational content. Most of the detected modes have been independently found in both datasets, and there is also evidence of variations in the mode excitations. We observed several prograde modes, together with a few axisymmetric and retrograde ones. In Fig. 1 a selected sample of modes detected in different stars is shown. They were related to FG Vir (Mantegazza, Poretti, \& Bossi 1994), X Cae (Mantegazza \& Poretti 1996; Mantegazza, Zerbi, \& Sacchi 1999), HD 101158 (Mantegazza 1997), BB Phe (Bossi, Mantegazza, \& Nuñez, 1998; Mantegazza \& Poretti 1999), BV Cir (Mantegazza, Poretti, \& Zerbi 1999). The plots show the behaviour of the phase of each mode across the line profile: a phase decreasing with wavelength (i.e. from left to right) indicates a prograde mode (i.e. a negative $m$ order). For each mode we give the observed frequency and the proposed identification (degree $\ell$ and azimuthal order $m$ ).

\section{References}

Bossi, M., Mantegazza, L., \& Nuñez, N. S. 1998, A\&A, 312, 855

Mantegazza, L. 1997, A\&A, 323, 845

Mantegazza, L. \& Poretti, E. 1996, A\&A, 312, 845

Mantegazza, L. \& Poretti, E. 1999, A\&A, 348, 139

Mantegazza, L., Poretti, E., \& Bossi, M. 1994, A\&A, 287, 95

Mantegazza, L., Poretti, E., \& Zerbi, F. M., 1999, in preparation

Mantegazza, L., Zerbi, F. M., \& Sacchi, A. 1999, A\&A, in press 


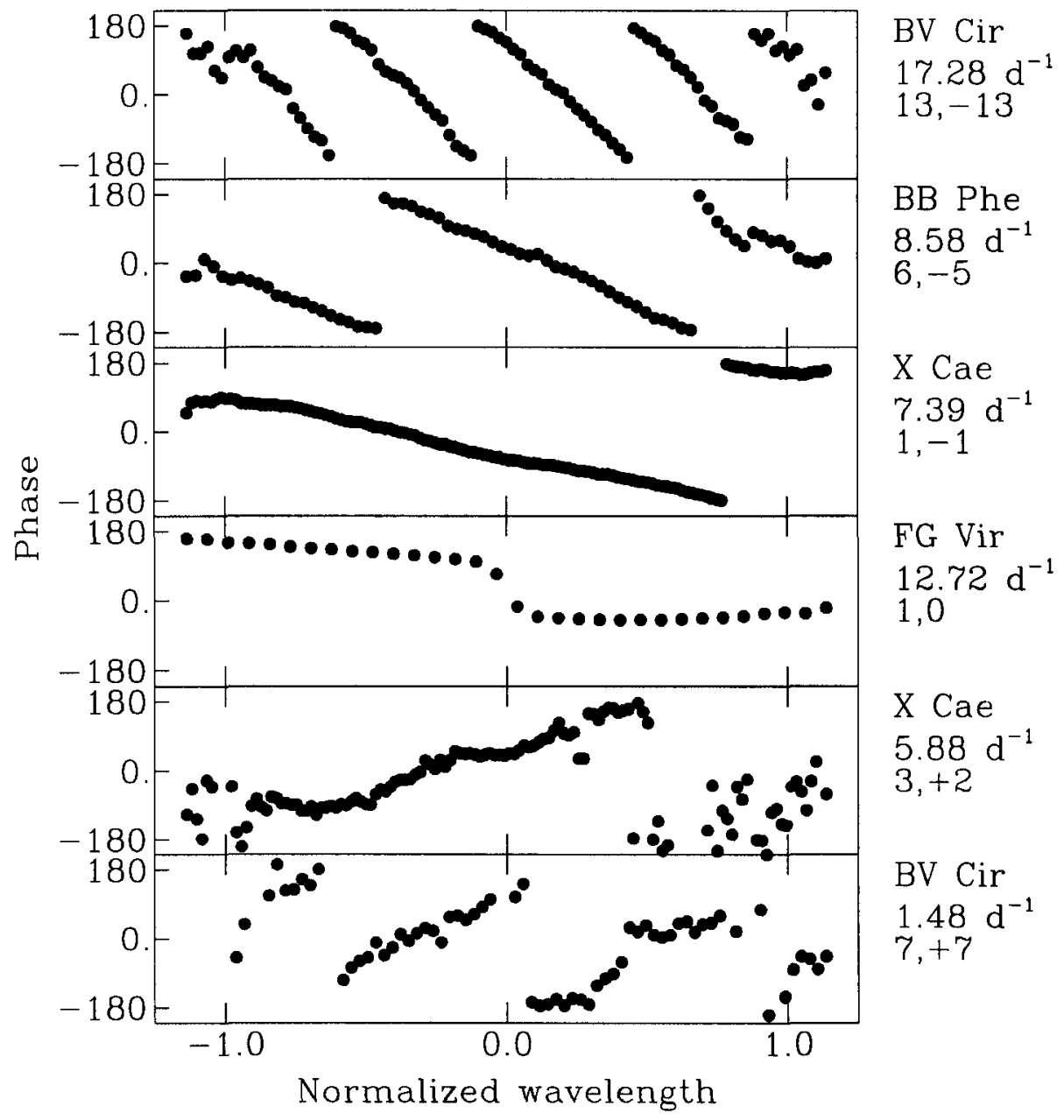

Figure 1. The zoo of nonradial modes observed in some $\delta$ Sct stars. On the right side the observed frequency and the related $\ell, m$ identification are reported. 